\title{
A Numerical Study of Fibrous Tissue Formation of Type I Collagen under Mechanical Stimuli by Reaction - Diffusion Equations with Motion of Fibroblasts
}

\author{
Takeshi Iwamoto*,1, Toshio Tsuta ${ }^{2}$ and Toshiyuki Sawa ${ }^{1}$ \\ ${ }^{1}$ Graduate School of Engineering, Hiroshima University, Japan \\ ${ }^{2}$ Faculty of Health Science, Hiroshima International University, Japan
}

\begin{abstract}
When the fibrous structure of collagen in soft tissue is adapted to an external load, its stiffness and topological characteristics are changed to achieve favorable function during the adaptation. Thus, a study of the formation of such fibrous tissue under mechanical stimuli is essential. To investigate the formation by the functional adaptation of fibrous tissue from a mechanical viewpoint, the vector of type I collagen fiber density is first defined by analogy to the dislocation density tensor. Then the mathematical model for the motion of a fibroblast and the reaction-diffusion equations for concentrations of the growth factors secreted from a fibroblast are generalized by considering the effect of mechanical load. After a multi-axial constitutive equation for the fibrous tissue is proposed, a finite element simulation of fibrous tissue formation by type I collagen fibers under mechanical stress is performed.
\end{abstract}

\section{INTRODUCTION}

The skin is the largest organ of the human body, and its weight reaches about $15 \%$ of body weight [1]. It carries out various functions such as moisture retention, body temperature regulation, perception, and it breathes to keep the body healthy [1]. At same time, it also plays a role of as a physical barrier between the external environment and the internal organs. Thus, the skin is mechanically stiff in order to protect internal organs not only from invasions of a foreign substance but also from the external load. Fig. (1) shows a schematic illustration of the hierarchical structure of skin on a face. At a finer length scale, various complicated structures with different components also appear as in the case with the heart, lungs, muscles, and the other organs. Specifically, a fibrous structure consisting of collagen fibers is stiffer at the micro-length scale which bridges the macro- to a nanolength scales, and this structure can support the mechanical load imposed.

At the same time, the fibrous structure of extracellular matrices such as collagen plays a key role in a series of diseases such as collagen disease involving the scleroderma, connective tissue disease, and autoimmune disease, which occur frequently and simultaneously [2]. One may think that these diseases cause an abnormal formation of extracellular matrices in tissues and make the organs harder as a result of a functional adaptation. The fibrous structure is established by complicated interactions between biological and mechanical factors. The macroscopic external load becomes a key factor. Unfortunately, medical treatment methods have not been developed yet since many unclear mechanisms have

*Address correspondence to these authors at the Department of Mechanical Systems Engineering, Graduate School of Engineering, Hiroshima University, 1-4-1 Kagamiyama, Higashi-Hiroshima, 739 - 8527, Japan; Tel: +8182-424-7576; Fax: +81-82-422-7193; E-mail: iwamotot@hiroshima-u.ac.jp been proposed from the viewpoints of pathology and etiology [2]. To clarify the mechanisms, it is necessary to extract not only the important biological factors but also the important mechanical factors at the microstructural level in the tissue and to determine if a relationship exists between fibrous tissue formation and functional adaptation on the basis of various governing factors.

Let us consider the closing of the eyes as shown in Fig. (1) as an example. In order to close the eyes, a macroscopic deformation of the skin realized by a muscle contraction makes a local reversible wrinkling near the edge of the eyes. If closing the eyes is repeated many times and/or aging takes place, part of the skin stiffens. As a result, wrinkling shifts from being reversible to being permanent. Similar to the diseases mentioned, this phenomenon is also one example of a functional adaptation. It can be understood that the skin can be adapted functionally by responding to a variety of mechanical environments. During the adaptation in this case, some events happen in the fibrous microstructure of collagen, which mainly supports the deformation caused by muscle contraction. One of the events is an increase in the size of a single collagen fiber, and the other may be a change in the topological characteristics of the structure such as an orientation, a number, and a configuration of a single fiber. As a result, a macroscopic property undergoes a change that depends on the topological characteristics of the microstructure. The mechanical load, which is induced by the deformation, is an important factor for the functional adaptation.

Up to now, many numerical and experimental studies considering mechanical stimuli have been carried out to reveal the formation of biological tissues such as skeletal muscle, blood vessels and bone. Tsubota et al. [3] simulated a change in a tissue in a trabecula, which is a microscopic structural element of bone, during functional adaptation by using a local nonuniform stress. Tezuka et al. [4] numerically examined the remodeling of bone by combining the 


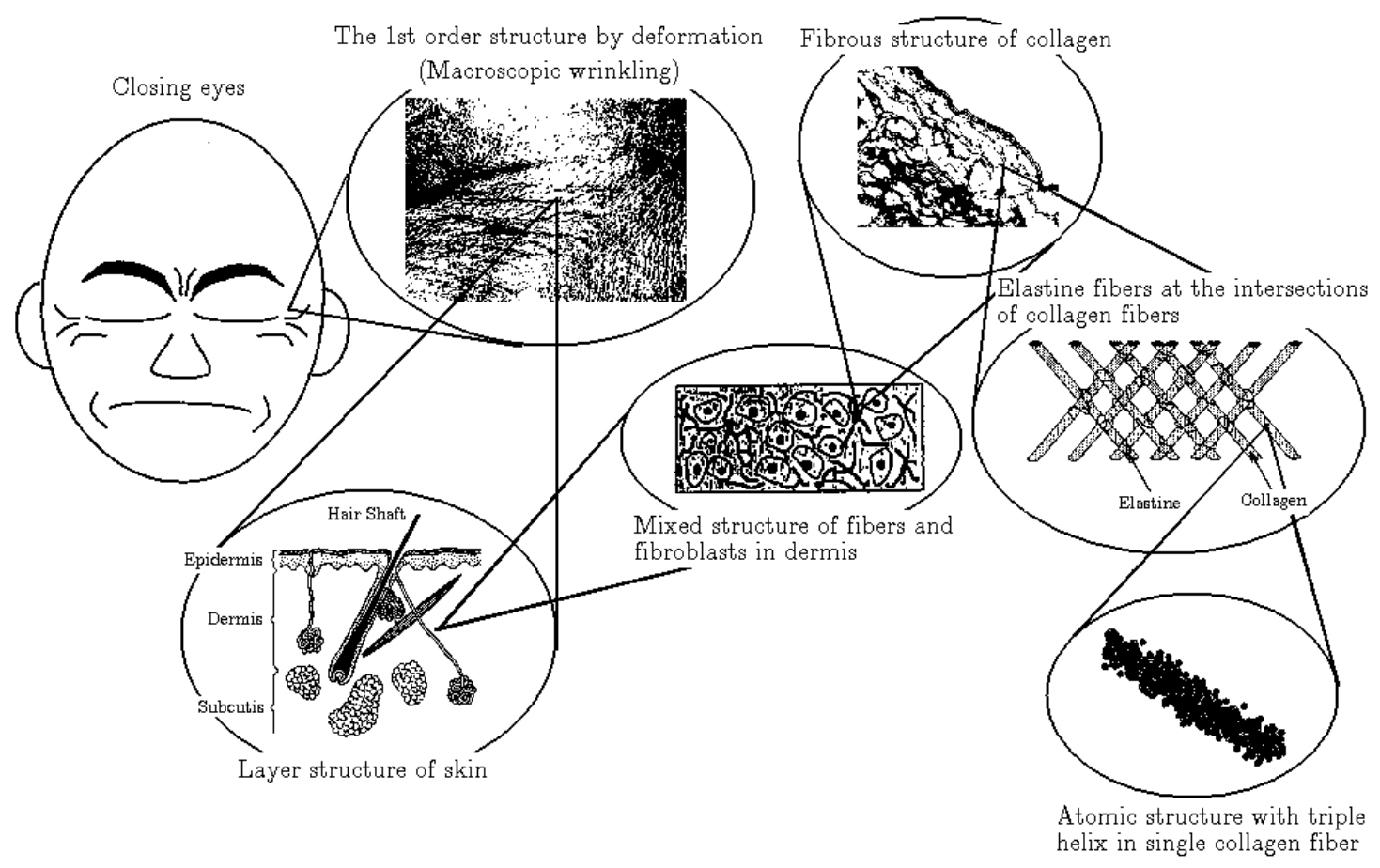

Fig. (1). A schematic illustration on a hierarchy of a structure in a skin under various length scales.

finite element method (FEM) and the reaction- diffusion equation of density in osteoblasts and osteoclasts. For a blood vessel, research has examined the relationship between an applied shear stress on a vessel wall and the orientation of endothrial cells in flowing blood [5]. For type I collagen, Dale et al. [6] proposed a group of reaction-diffusion equations for governing factors in the formation of fibrous tissue by collagen. Dallon et al. [7] studied a change in orientation of fibrous tissues with the motion of a fibroblast which deals with the assumption of a point-like cell. On the other hand, in order to study functions of a fibroblast such as recognition of and adaptation to mechanical load, experimental work has examined changes in a mechanical property of test piece made from a collagen gel inside of which fibroblasts are cultured, and then the change in the configuration of the fibroblast in the test piece is observed under stress stimuli [8], [9]. However, neither a clarification of the mechanism of formation of tissue nor the functional adaptation corresponding to an external load has been investigated sufficiently in the research described. To achieve this sizable goal, not only an excellent experiment but also a numerical simulation based on a sophisticated model is strongly required.

In this paper, we present our understanding of functional adaptation by the formation of tissue in skin from a mechanical viewpoint. We studied a fibrous tissue of type I collagen, which is the principal component of the dermis. A vector of collagen fiber density is defined by its similarity with the dislocation density tensor [10] used frequently in metallurgy. A group of reaction-diffusion equations proposed by Dale et al. [6] and the models proposed by Dallon et al. [7] are extended to consider not only cell migration and chemotaxis but also the effects of the mechanical stimuli based on a strain energy and a principal stress. In addition, the rate form of a constitutive equation at a local region for collagen fibrous tissue is derived using a volume average of a stress rate. The constitutive equation is a function of the vector of collagen fiber density. Finally, influences of a macroscopic tensile stretching on the formation of fibrous tissue are examined by a coupled computational analysis of the proposed model by FEM [11].

\section{MATHEMATICAL MODEL OF THE FORMATION OF FIBROUS TISSUE}

\section{Definition of Vector of Collagen Fiber Density and its Evolution Equation}

A vector of collagen fiber density is defined by using its similarity with the dislocation density tensor frequently employed in metallurgy. According to the definition, the vector indicates the total length of collagen fibers per unit volume [10]. Its physical meaning becomes the total number of collagen fibers that pass through a unit area; the vector has unit of $1 / \mathrm{m}^{2}$. Fig. (2) shows a schematic representation of a collagen fiber. The collagen fiber can be written using a line; however, the molecular structure is a complicated triple helix observed by magnifying part of a line-like collagen fiber. As shown in Fig. (2), the wavelength (like the pitch of a screw) vector of the helical structure in the molecules of $(a)$ th collagen fiber is assumed to be $\vec{b}^{(a)}$. Let us consider the situation in which the $(a)$ th collagen fiber with a unit vector $\vec{v}^{(a)}$ in the longitudinal direction corresponds to a unit normal vector $\vec{n}$ on an infinitesimal area $s$ including a point $\vec{x}$ when the $(a)$ th fiber passes through $s$. From this physical meaning, the tensor of the collagen fiber density $\vec{\gamma}$ can be defined as follows: 
$\vec{\gamma}=\frac{1}{S} \sum_{a=1}^{N_{c}} \vec{v}^{(a)} \otimes \vec{b}^{(a)}$.

Here, $N_{c}$ is the total number of collagen fibers which pass through $S$. Since this expression may mean an average, the limit of Eq. (1) is taken, and the next expression defined at an arbitrary point $\vec{x}$ is used as

$\vec{\gamma}=\lim _{s \rightarrow 0} \frac{1}{S} \sum_{a=1}^{N_{c}} \vec{v}^{(a)} \otimes \vec{b}^{(a)}$.

However, there is a different point from the case of dislocation density; namely, that $\vec{b}^{(a)}$ and $\vec{v}^{(a)}$ have the same direction. From this fact, $\vec{\gamma}$ can be expressed using a contraction from the tensor to the vector. This is the definition of a vector of collagen fiber density, and it is defined mathematically as follows:

$\vec{c}=\lim _{s \rightarrow 0} \frac{1}{S} \sum_{a=1}^{N_{c}}\left\|\vec{b}^{(a)}\right\| \vec{v}^{(a)}=c_{i} \vec{n}_{i}$.

Here, $\|\bullet\|$ shows the magnitude of vector $\bullet$. The collagen fibrous tissue can be dealt with by the continuous vector field using $\vec{c}$.

On the other hand, the evolution equation of $\vec{c}$ can be modeled as follows. $\vec{c}$ has both magnitude and direction. The evolution equations can be considered separately. The direction indicates the statistical fact that collagen fibers occupy a domain at a certain time.

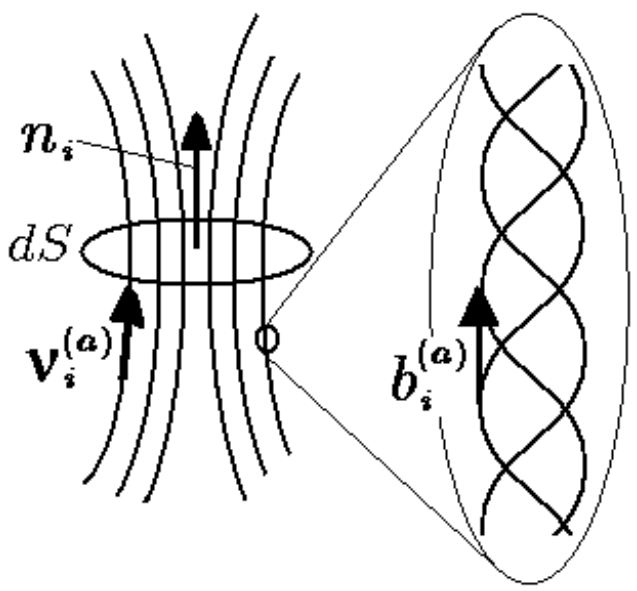

Fig. (2). Definition of the vector of collagen fiber density.

It is assumed that the direction to which the fibroblasts move affects the direction of $\vec{c}$, as discussed in Dallon et al. [7]. In addition, since the oriented structure of a trabecula depends on the direction of the principal stress in a biological tissue such as bone [12], we choose the assumption that a similar phenomenon occurs in the tissue of skin, and the model proposed by Dallon et al. [7] can be generalized as

$$
\begin{aligned}
& \dot{\theta}(\vec{x}, t)=v\|\vec{f}\| \sin (\alpha-\theta) \\
& +\lambda\|\vec{\sigma}\| \sin (\beta-\theta) .
\end{aligned}
$$

$\theta$ indicates the orientation angle of $\vec{c}, \vec{\sigma}$ and $\beta$ are the principal stress vector and its direction angle, and $\alpha$ is the direction angle of $\vec{f}$ which we describe later. $v$ is a parameter determined by characteristics of both the fibroblasts and the collagen fiber, and it shows that the fibroblast may change the direction of collagen fiber. $\lambda$ is a newlyintroduced parameter that shows that $\vec{\sigma}$ may change the direction of collagen fiber just as well as $v$.

Obeying the derivation of the model proposed by Dallon et al. [7], $\vec{f}$ indicates the influence which fibroblasts move as a group, and it can be expressed using the weight function $w_{i}$ as

$$
\begin{aligned}
& \vec{f}(\vec{x}, t)=\sum_{i=1}^{N} w_{i}(\vec{x}, t) \frac{\dot{\vec{f}}^{i}(t-\tau)}{\left\|\dot{\vec{f}}^{i}(t-\tau)\right\|}, \\
& w_{i}(\vec{x}, t)=\prod_{j=1}^{D} a_{j}^{i}(\vec{x}, t), \\
& a_{j}^{i}(\vec{x}, t)=\max \left(1-\frac{\left|f_{j}^{i}(t)-x_{j}\right|}{L}, 0\right) .
\end{aligned}
$$

Here, $N$ is the number of fibroblasts, $D$ is the dimension of space, the superscript $i$ is the number to identify the fibroblast, and $L$ is the characteristic length of the effective domain of $w_{i} . \tau$ is the time delay to change the direction in which the fibroblast moves after it undergoes any stimulation from the surrounding environment. $\vec{f}^{i}$ indicates the position vector of the fibroblast, and its details are described in the next section.

The magnitude $c_{I}=\|\vec{c}\|$ of $\vec{c}$ shows the concentration of collagen fiber, and it strongly depends on both the composition of the extracellular matrices and the biochemical environments [6]. Chemical substances are secreted collectively by surrounding fibroblasts. As the result, the formation of tissue is promoted [7]. Here, the time evolution of the concentration of the collagen fiber $c_{I}$ is expressed by considering both influences as

$\dot{c}_{I}=\left(A_{1} p_{I} e_{I I}-A_{2} s_{I} c_{I}\right) \sum_{i=1}^{N} w_{i}(\vec{x}, t)$.

Here, $A_{1}$ and $A_{2}$ are the coefficients of the reaction kinetics, $p_{I}$ is the concentration of pro-collagen which is used to synthesize type I collagen fiber, $e_{I I}$ is the concentration of enzyme which activates pro-collagen, and $s_{I}$ is the concentration of collagenase which degrade type I collagen fiber. The first term in the round bracket on the right hand side of Eq. (6) indicates that the production of collagen obeys zeroth order reaction kinetics; the second term is the 
degradation of $c_{I}$ by a first order reaction. The summation of $w_{i}$ means the contribution of all fibroblasts inside the domain with $L$ to produce $c_{I}$.

The reaction rate shown in the above equation markedly depends on temperature and pressure [13]. Since the Gibbs free energy is a function of the strain energy stored in deformable media, it becomes possible to describe the relationship between the coefficients $A_{i}$ in the equation, the absolute temperature $T$, and the strain energy $W$ as

$$
A_{i}=k_{i} \exp \left(\frac{W}{k_{B} T}\right)
$$

Here, $k_{B}$ is the Boltzmann constant and $k_{i}$ is the coefficients of reaction kinetics when the strain energy is not considered.

\section{Motion of Fibroblasts}

Fibroblast can move in any direction after receiving stimuli from the extracellular matrices [7]. Additionally, it can be assumed that the collective moving velocity of fibroblasts is similar to the basic concept of a convectiondiffusion equation for a molecule, and the migration of the fibroblasts can be quantified using the transportation equation for the collective motion of the fibroblasts [14]. As done by Dallon et al. [7], these factors can be included using a position vector of the fibroblast $\vec{f}^{i}=\left(f_{1}^{i}, f_{2}^{i}, f_{3}^{i}\right)$, and its time evolution can be expressed as

$$
\begin{aligned}
& \dot{\vec{f}}^{i}=(1-\rho-\gamma-\delta) \vec{c}\left(\vec{f}^{i}, t\right) \\
& +\rho \frac{\dot{\bar{f}}^{i}(t-\tau)}{\left\|\dot{\bar{f}}^{i}(t-\tau)\right\|}-\gamma \frac{\vec{M}\left(\vec{f}^{i}, t-\tau\right)}{\left\|\vec{M}\left(\vec{f}^{i}, t-\tau\right)\right\|} \\
& +\delta \frac{\vec{N}\left(\vec{f}^{i}, t-\tau\right)}{\left\|\vec{N}\left(\vec{f}^{i}, t-\tau\right)\right\|},
\end{aligned}
$$

where $\rho, \gamma$, and $\delta$ are positive constants. Moreover, the moving velocities for the migration $\vec{M}\left(\vec{f}^{i}, t-\tau\right)$ and chemotaxis $\vec{N}\left(\vec{f}^{i}, t-\tau\right)$ of the fibroblasts can be shown as [14]

$\vec{M}=\vec{\mu} \bullet \nabla f, \vec{N}=\ddot{\chi} \bullet \nabla A$,

where $f$ and $A$ are the concentrations of the fibroblasts and the attracting factor. The symbol - means the inner product of the tensor. $\vec{\mu}$ is the coefficient for the migration of the fibroblast and is similar to the diffusion coefficient. $\vec{\chi}$ is a coefficient of chemotaxis and is the function of $A$ [14]. Chemotaxis is produced by from the spatial distribution of a number of complexes composed of a receptor and an attractive factor over the total the circumferential length of the fibroblast [14]. The third term on the right hand side in Eq. (9) shows the dispersive motion of the fibroblasts along with a spatial gradient for the concentration of the fibroblasts, and the fourth term indicates a convection velocity for the motion of the fibroblasts through a spatial gradient of $A$ [14].

\section{A Group of Reaction-Diffusion Equations for Fibro- blasts-Derived Substances}

The formation of tissue is accomplished through the complicated interactions between various factors, substances, and cells in the tissue. Since it is quite difficult to consider all these factors in detail, a simplified model is effective. Obeying the ideas of Dale et al. [6], type I and III collagen are considered because they are major ingredients in the dermis. The biochemical reactions of a production system and a degradation system are shown in Fig. (3). According to this figure, the evolution equation for each chemical substance can be formulated as follows.

The fibroblast is the principal cell of the dermis and is sparsely dispersed in the extracellular matrices. In the case of a low concentration of TGF- $\beta$, a group of fibroblast exponentially increases and its proliferation at saturated in its highest concentration. The model can be expressed as

$$
\dot{f}=\left(A_{3}+A_{4} \beta_{I}+A_{5} \beta_{I I I}\right) f\left(1-\frac{f}{k_{1}}\right)-A_{4} f
$$

Here, $\beta_{I}$ and $\beta_{I I I}$ are the concentrations of the active TGF- $\beta$ which act to produce type I and III collagen fibers, respectively. The second term indicates apoptosis of the fibroblast.

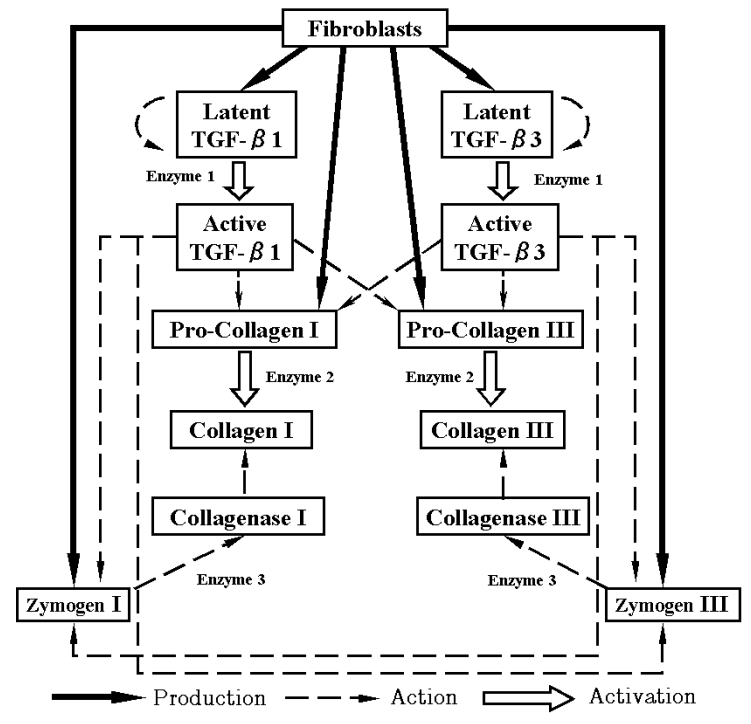

Fig. (3). A schematic illustration of correlations in biochemical reactions on type I collagen production.

TGF- $\beta$ is dealt with as a growth factor, which helps collagen production. The fibroblast is stimulated with any type of TGF- $\beta$, and it secretes latent TGF- $\beta$. The latent TGF- $\beta$ freely diffuses, it barely increases to infinity, and it undergoes the autocrine mechanism (self-activation). Therefore, TGF- $\beta$ increases by self-secretion. These phenomena can be expressed as 


$$
\begin{aligned}
& \dot{\ell}_{I}=D_{2} \nabla^{2} \ell_{I}+\frac{A_{6} f \ell_{I}}{1+A_{7} \ell_{I I I}+A_{8} \ell_{I}} \\
& -A_{9} \ell_{I}-A_{10} e_{I} \ell_{I}, \\
& \dot{\ell}_{I I I}=D_{3} \nabla^{2} \ell_{I I I}+\frac{A_{11} f \ell_{I I I}}{1+A_{12} \ell_{I I I}} \\
& -A_{13} \ell_{I I I}-A_{14} e_{I} \ell_{I I I},
\end{aligned}
$$

where $\ell_{I}$ and $\ell_{I I I}$ are the concentration of latent TGF- $\beta$

Because the active TGF- $\beta$ also diffuses freely similarly to the latent TGF- $\beta$, it can be modeled by the diffusion equation of Fick's law to move chemically

$$
\begin{aligned}
& \dot{\beta}_{I}=D_{4} \nabla^{2} \beta_{I}+A_{15} e_{I} \ell_{I}-A_{16} \beta_{I}, \\
& \dot{\beta}_{I I I}=D_{5} \nabla^{2} \beta_{I I I}+A_{17} e_{I} \ell_{I I I}-A_{18} \beta_{I I I} .
\end{aligned}
$$

Pro-collagen and collagenase are considered primary factors in the production system and a degradation system of collagen, respectively. Pro-collagen is synthesized by the fibroblasts, and the synthesis is stimulated by TGF- $\beta$. Procollagen fiber barely diffuses in the tissue. It can be assumed that it is secreted constantly from fibroblasts when other chemical substances have a low concentration. It can be modeled that pro-collagen dies naturally in accordance with a first order reaction, assuming that the longevity of a procollagen fiber is constant

$$
\begin{aligned}
& \dot{p}_{I}=\left(A_{25}+A_{26} \beta_{I}+A_{27} \beta_{I I I}\right) f \\
& -A_{28} p_{I}-A_{29} e_{I I} p_{I}, \\
& \dot{p}_{I I I}=\left(A_{30}+A_{31} \beta_{I}+A_{32} \beta_{I I I}\right) f \\
& -A_{33} p_{I I I}-A_{34} e_{I I} p_{I I I},
\end{aligned}
$$

where $p_{I I I}$ is the concentration of pro-collagen which acts on the production of type III collagen fiber. Collagenase is an active zymogen; it actively binds to collagen, and the fiber is thereby destroyed. The natural decrease of collagenase is modeled by a first order reaction as

$\dot{s}_{I}=A_{48} z_{I} e_{I I I}-A_{49} s_{I}$,

$\dot{s}_{I I I}=A_{50} z_{I I I} e_{I I I}-A_{51} s_{I I I}$,

where $z_{I}$ and $z_{I I I}$ are the concentrations of zymogens which assist the degradation of type I and III collagen fibers, respectively. $S_{I I I}$ is the concentration of collagenase which degrades type III collagen fiber.

A zymogen is secreted from the fibroblasts as an inactive collagenase. It has no ability to degrade collagen until it is activated. Diffusion of zymogens is not well-characterized, and they are synthesized and secreted as the fibroblasts move. However, their secretion is suppressed by the presence of active TGF- $\beta$. The suppression is assumed to be linear; in addition, the natural death of zymogen takes place and obeys the first order reaction kinetics, and a zymogen changes into a collagenase after it is activated with a specific enzyme $e_{I I I}$ as

$$
\begin{aligned}
& \dot{z}_{I}=\frac{A_{38}}{1+A_{39} \beta_{I}+A_{40} \beta_{I I I}} f c_{I} \\
& -A_{41} z_{I}-A_{42} e_{I I I} z_{I}, \\
& \dot{z}_{I I I}=\frac{A_{43}}{1+A_{44} \beta_{I}+A_{45} \beta_{I I I}} f c_{I I I} \\
& -A_{46} z_{I I I}-A_{47} e_{I I I} z_{I I I},
\end{aligned}
$$

where $c_{I I I}$ is the concentration of type III collagen fiber.

Type III collagen is also a protein with a threefold helical structure, and it is formed when type III pro-collagen is activated. The following evolution equation can be formulated under the assumption of gradual and linear degradation of type III collagen by type III collagenase

$\dot{c}_{I I I}=A_{36} p_{I I I} e_{I I}-A_{37} s_{I I I} c_{I I I}$.

It can be assumed that this evolution equation does not include a spatial gradient because no experimental facts are available on the diffusion of type III collagen.

In the tissue formation process, a variety of active growth factors interact. However, the interactions are numerous, complex, and many points are still unclear. To simplify the model, only the specific enzymes $e_{I}, e_{I I}$, and $e_{I I I}$, which activate the latent TGF- $\beta$, pro-collagen, and zymogen, are considered. Their evolution equations can be expressed as

$$
\begin{aligned}
& \dot{e}_{I}=-e_{I}\left(A_{19} \ell_{I}+A_{20} \ell_{I I I}\right), \\
& \dot{e}_{I I}=-e_{I I}\left(A_{21} p_{I}+A_{22} p_{I I I}\right), \\
& \dot{e}_{I I I}=-e_{I I I}\left(A_{23} z_{I}+A_{24} z_{I I I}\right),
\end{aligned}
$$

where it can be assumed that an increase in the concentration of enzyme is spatially independent.

\section{Cell Adhesion}

The fibroblast is an anchorage-dependent cell. As an anchorage-dependent cell, the fibroblast plays important roles in the synthesis and degradation of chemical components and controls self-proliferation after recognizing its environment by adhering to the extracellular matrices [15]. Therefore, it is necessary to consider cell adhesion to the extracellular matrices. When the following criterion is satisfied as a adhesive condition to the extra cellular matrices, it is assumed $\dot{\vec{f}}^{i}=0$ and $c_{I}>c_{I C}$,

when

$$
\left|\vec{f}^{i}(t)-\vec{x}(t)\right|<r,
$$

where $r$ and $c_{I C}$ are constants for a threshold. Originally, the production of adhesive protein by the fibroblast such as a 
fibronectin and laminin, which promote cell adhesion in the biosyntheses of type I collagen must be considered. Here, the effect is on cell adhesion considered and it can be omitted to simplify the model.

\section{Evaluation of Local and Global Stress in Fibrous Tissue}

To employ the updated Lagrangian method and the finite element equation based on the rate form of the principle of virtual work, a rate form of a constitutive equation for a given collagen fiber density is derived. First, the following uniaxial constitutive equation experimentally obtained by Haut et al. [16] is employed for the relationship of nonlinear uniaxial stress $\sigma$ - strain $\mathcal{E}$ and strain rate $\dot{\mathcal{E}}$ for a single collagen fiber

$\sigma(\varepsilon, \dot{\varepsilon})=\left[1+\mu\left\{\ln \left(\frac{\varepsilon}{\dot{\varepsilon}}\right)-\frac{3}{2}\right\}\right] \frac{E}{2} \varepsilon^{2}$,

where $\mu$ and $E$ are the material constants, respectively.

The uniaxial stress $\sigma$ in Eq. (19) is assumed to be one component of the stress tensor $\vec{\sigma}^{(a)}$ on the coordinate system placed on the $(a)$ th collagen fiber in the microscopic area. In this case, all other components of $\vec{\sigma}^{(a)}$ are zero. In addition, a structural tensor $\vec{Q}^{(a)}$ to express the direction of the $(a)$ th collagen fiber is introduced. Using $\vec{\sigma}^{(a)}$ and $\vec{Q}^{(a)}$, the Jaumann rate of the second Piola-Kirchhoff stress $\nabla(a)$

$\vec{S}$ of the $(a)$ th collagen fiber on the reference frame arranged in the microstructure can be written in the form of a coordinate transformation as

$\stackrel{\nabla}{S}^{(a)}=\vec{Q}^{(a)^{T}} \dot{\vec{\sigma}}^{(a)} \vec{Q}^{(a)}, \vec{Q}^{(a)}=\vec{v}^{(a)} \otimes \vec{v}^{(a)}$.

It is assumed that the macroscopic stress rate with objectivity $\stackrel{\nabla}{\stackrel{\nabla}{\Sigma}}$ can be calculated from a volume average of the microscopic stress rate $\vec{S}$ as

$\stackrel{\nabla}{\Sigma}=\lim _{v \rightarrow 0} \frac{1}{v} \int_{v} \stackrel{\nabla^{(a)}}{\vec{S}} d v$

$=\lim _{v \rightarrow 0} \frac{1}{v} \int_{v} \vec{Q}^{(a)^{T}} \dot{\vec{\sigma}}^{(a)} \vec{Q}^{(a)} d v$

Here, $v$ is the infinitesimal representative volume element considered in a body.

By considering a finite $v$, we can employ a summation with respect to a further infinitesimal volume $d v$ on $v$ instead the integral as

$\stackrel{\nabla}{\Sigma}=\lim _{v \rightarrow 0} \frac{1}{v} \sum_{(a)} \vec{Q}^{(a)}{ }^{T} \dot{\vec{\sigma}}^{(a)} \vec{Q}^{(a)} \Delta \ell^{(a)}$,

where $\Delta \ell^{(a)}$ is the infinitesimal length of the (a)th collagen fiber.
The following mixture rules can be obtained if $\vec{Q}^{(a)^{T}} \dot{\vec{\sigma}}^{(a)} \vec{Q}^{(a)}$ becomes uniform about each fiber at the limit as

$\stackrel{\nabla}{\Sigma}=\vec{Q}^{T} \dot{\widetilde{\sigma}} \vec{Q} c_{I}$, and $\vec{Q}=\vec{n} \otimes \vec{n}$.

Moreover, if one component of the stretching tensor $\vec{d}$ corresponds to the uniaxial strain rate $\dot{\mathcal{E}}$ in Eq. (19), the macroscopic constitutive equation as a function of collagen fiber density and orientation angle can be obtained as a form of

$\stackrel{\nabla}{\Sigma}=\vec{D}^{(a)}\left(c_{I}, \theta\right) \vec{d}$.

\section{FINITE ELEMENT FORMULATIONS, COMPUTAT- IONAL MODEL AND CONDITIONS}

Roughly speaking, there are two fields to be solved in the problem. One is the stress field, and the other is the diffusion field. To obtain the solution of the stress field, the rate form of constitutive equation (24) is introduced into the rate form of the principle of virtual work, and the computational simulation is performed by FEM. The rate form of the principle of virtual work in the finite deformation regime can be formulated for the current configuration as

$\int_{\Omega}\left(\dot{\vec{\Sigma}}+\vec{\sigma} \cdot \frac{\partial \vec{v}}{\partial \vec{x}}\right) \cdots \frac{\partial \delta \vec{v}}{\partial \vec{x}} d \Omega=\int_{S_{t}} \dot{\vec{F}} \cdot \delta \vec{v} d S$,

where $\vec{v}$ is the velocity field, $\vec{\Sigma}$ is the second PiolaKirchhoff stress tensor, $\vec{\sigma}$ is the Cauchy stress tensor, $\vec{F}$ is the surface tension vector, and $\Omega$ and $S$ are the volume and surface of the deformable body being considered, respectively. The symbol $\bullet$ means the inner product of the tensors. The velocity $\vec{v}$ and its spatial derivative can be discretized by the shape function matrix $\mathbf{N}$ and the nodal quantity of the velocity $\dot{\mathbf{d}}$ :

$\vec{v}=\mathbf{N} \dot{\mathbf{d}}, \mathbf{g}=\mathbf{B} \dot{\mathbf{d}}$, and $\mathbf{q}=\mathbf{E} \dot{\mathbf{d}}$,

where bold face indicates the matrices and vectors of nodal value. $\mathbf{g}$ and $\mathbf{q}$ are the nodal vectors corresponding to $\vec{d}$ and the velocity gradient tensor, respectively. After rearrangement obeying the conventional $\mathrm{FE}$ formulation, we obtain the following equation:

$\mathbf{K} \dot{\mathbf{d}}=\dot{\mathbf{f}}$,

$\mathbf{K}=\int_{\Omega}\left[\mathbf{B}^{T}(\mathbf{D}-\mathbf{G}) \mathbf{B}+\mathbf{E}^{T} \mathbf{Q E}\right] d \Omega$,

$\dot{\mathbf{f}}=\int_{S_{t}} \mathbf{N}^{T} \dot{\mathbf{F}} d S$

where $\mathbf{D}$ is the matrix of the constitutive tensor in Eq. (24), and $\mathbf{G}$ and $\mathbf{Q}$ are the matrices derived from the consideration of the finite deformation.

A group of the reaction-diffusion equations shown in Eq. (6), and Eqs. (10) to (17) are strongly coupled to each other. However, it requires too high a computational cost to calcu- 
late the solutions. In order to reduce the huge cost, the weak coupling assumption is introduced [11]. In this assumption, the time step must be shortened as much as possible, and instead each equation can be solved sequentially. The solutions of the equations, except for Eqs. (11) and (12), can be obtained using the finite difference method for only the time integration since the spatial derivative is not included. Here, at least four partial differential equations expressed in Eqs. (11) and (12) are solved by FEM. For simplicity of explanation in the FE formulation based on the reaction-diffusion equations discussed, only one equation is formulated as follows. According to weighted residual method [11], the weak form of governing equation Eq. (6) can be formulated as follows:

$\int_{\Omega}\left(\dot{\beta}_{I}-D_{4} \nabla^{2} \beta_{I}+A_{15} e_{I} l_{I}-A_{16} \beta_{I}\right) \delta \beta_{I} d \Omega=0$,

The concentration $\beta_{I}$ and its time derivative can be discretized by the shape function matrix $\overline{\mathbf{N}}$ and the nodal quantity of the concentration $\mathbf{b}$ :

$\beta_{I}=\overline{\mathbf{N}} \mathbf{b}, \dot{\beta}_{I}=\overline{\mathbf{N}} \dot{\mathbf{b}}$,

and $\overline{\mathbf{g}}=\nabla \beta_{I}=\overline{\mathbf{B}} \mathbf{b}$.

After rearrangement obeying the conventional FE formulation, we obtain the following equation:

$\overline{\mathbf{C}} \dot{\mathbf{b}}+\overline{\mathbf{K}} \mathbf{b}=\overline{\mathbf{f}}$,

$\overline{\mathbf{C}}=\int_{\Omega} \overline{\mathbf{N}}^{T} \overline{\mathbf{N}} d \Omega$,

$\overline{\mathbf{K}}=\int_{\Omega}\left(D_{4} \overline{\mathbf{B}}^{T} \overline{\mathbf{B}}-A_{16} \overline{\mathbf{N}}^{T} \overline{\mathbf{N}}\right) d \Omega$,

and, $\overline{\mathbf{f}}=\int_{\Omega} \overline{\mathbf{N}}^{T} A_{15} e_{I} l_{I} d \Omega$.

Fig. (4) shows the computational model for the twodimensional region. The computational region is a rectangular area with an edge length of $0.05 \mathrm{~mm}$, and it is divided into ten isoparametric quadratic elements with eight nodes in the directions of $x$ and $y$, respectively. As an initial condition, a total of 25 fibroblasts are arranged in this area using random numbers as shown in figure. The parameters uses in all equations are the values published in Refs. [5], [6], and [15]. When the tensile deformation is given, the displacement rate in the $x$ direction is given to the right side, and other constraint conditions are as shown in the figure.

\section{COMPUTATIONAL RESULTS AND DISCUSSIONS}

In order to discuss the computational results of fibrous tissue formation without any mechanical stimulations at first, Fig. (5) shows (a) the electron micrograph of type I collagen fibrous tissue taken from [17], (b) the distribution in the vector of the collagen fiber density, (c) the distribution in collagen fiber density, and (d) a histogram of the direction in the vector of the collagen fiber density. Figs. (5b) to (5d) are obtained from the computation. The fibroblasts are shown as solid circles in Fig. (5b). From Fig. (5b), the network structure can be obtained by computation, and it is quite similar to the actual tissue in the photograph shown in Fig. (5a). It can be observed that the fiber is oriented as a vortex at the center

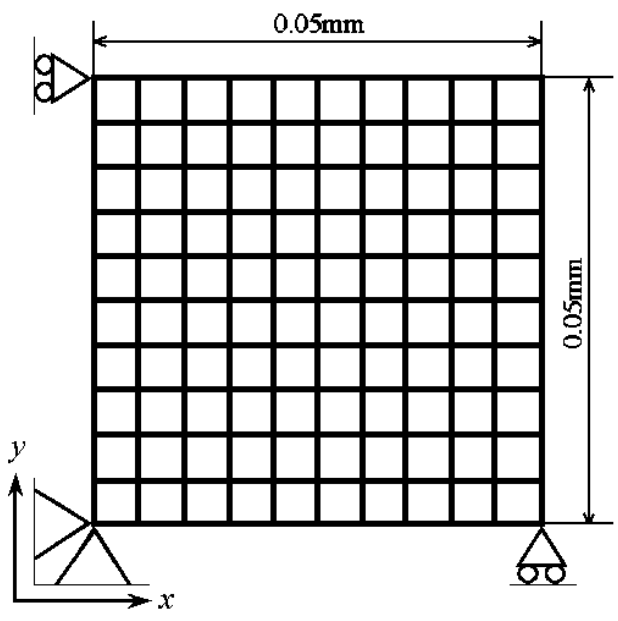

(a)

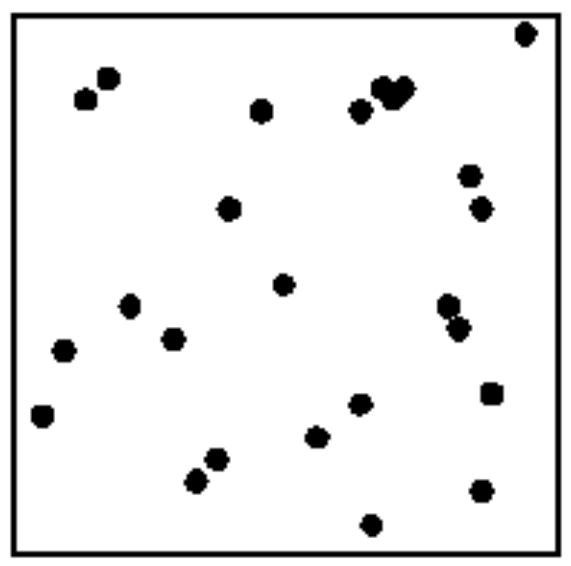

(b)

Fig. (4). (a) Finite element model in 2D rectangular space and (b) initial configuration of 25 fibroblasts.

of the area, and the fiber is synthesized over almost the entire area. Corresponding to this result, the region where the fiber density becomes higher can be seen from Fig. (5c) at the center of the area, and the region of a low fiber density can be observed in the vicinity of the boundary. As shown in Fig. (5d), the frequency in the direction of the collagen fiber density is counted in each $45 \mathrm{deg}$ range. The orientation angle of 0 deg indicates that the fiber is parallel to the $y$ axis, and it is positive in the clockwise direction. In Fig. (5d), the fibers mainly orient to 45 and $135 \mathrm{deg}$, however, the data indicate that the frequency without the deformation is comparatively uniform.

Fig. (6) shows the distribution in the vector of the collagen fiber density under a strain of (a) $10 \%$ and (b) $20 \%$. From Figs. (6a) and (6b), the basic features can be observed as well as the case without deformation even if mechanical stimulation is given. The vector of the fiber density with an orientation angle near 135 deg appears at the left part of the area, and the vector in the vertical direction can be observed on the right side as shown in Fig. (6a). Many fibers are generated from the center to the upper part in the area. With the promotion of deformation, the fibers show quite complicated orientation, such as the vortex-like configuration at the cen- 


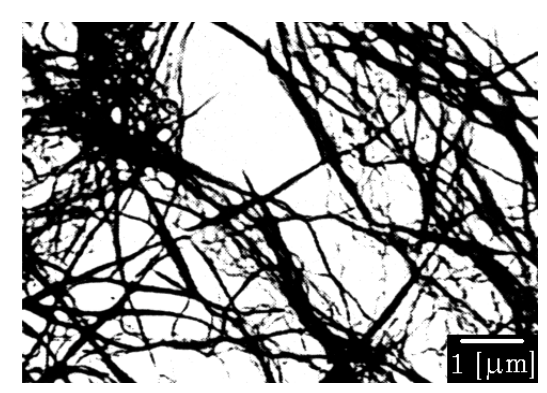

(a) Observation of micrograph

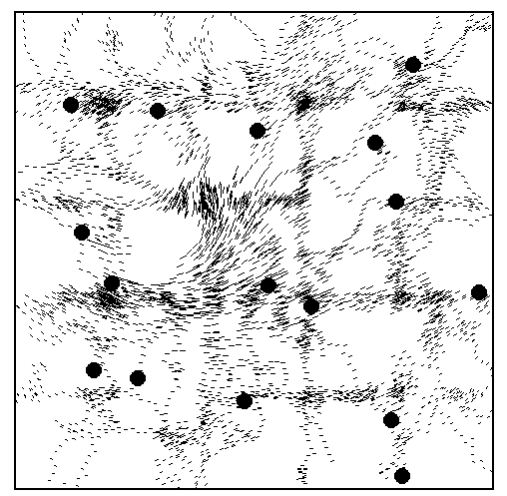

(b) Configuration of density of collagen fiber

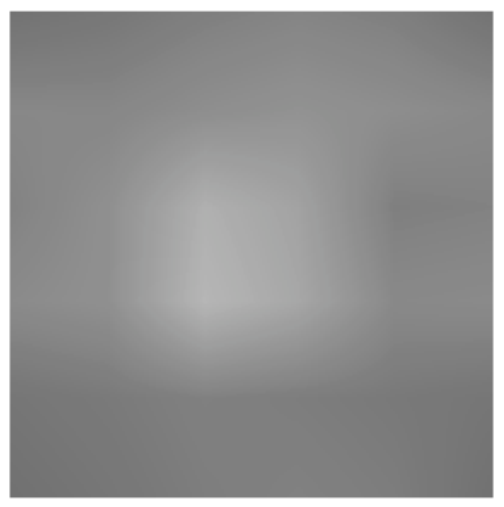

(c) Distibution of density of collagen fiber

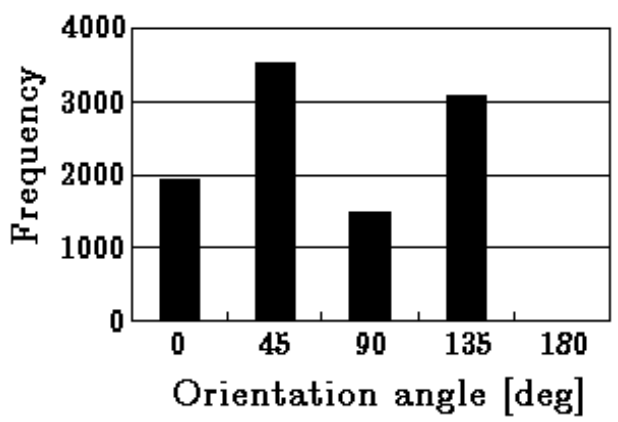

(d) Histogram of orientation angle

Fig. (5). Computational results on tissue formation of collagen fiber without mechanical stimulation.

ter of the area shown in Fig. (6b). The area where the fiber is synthesized voluminously moves to the center. The region where the fiber density becomes zero moves to the lower side and to the left of the model. In addition, the bigger fibrous structure, which consists of the vortex and network, is formed at the center of the model. Focusing on the fibroblasts, the fibroblasts are mutually adjacent compared with the case without deformation although the configuration of fibroblasts is random throughout the entire area. The remarkable tendency appears that the fibroblasts initially approach each other near the center of the model, and then the intercellular distance shortens with the progress of the deformation. Figs. (6c) and (6d) show histograms of the orientation angle in the vector of the collagen fiber density. From Fig. (6c), many fibers have the horizontal direction at a strain of $10 \%$. However, the frequency in the direction of $45 \mathrm{deg}$ is the highest after further deformation as shown in Fig. (6d). From the histograms shown in Figs. (5 and 6), a comparatively uniform distribution of the orientation of fibers can be observed in the case without deformation, and then a bias in the distribution in either direction of 45 or $135 \mathrm{deg}$ can be seen once the deformation is given. Concretely speaking, the fibers orient to strongly in a parallel direction against the tensile axis in the initial stage of deformation, and then they collectively rotate to the direction of maxi-mum shear stress. It can be assumed that the fibers collectively orient at a certain specific direction when the deformation is given, and this phenomenon is the adaptation against the deformation.

Fig. (7) shows the distribution of (a) the collagen fiber density and (b) the normal stress component in the tensile direction when a tensile strain of $20 \%$ is applied. From Fig. (7a), it can be seen that the fiber density has a higher value near the center of the area as in the case without deformation shown in Fig. (5c). However, the area with the higher fiber density expands by comparison with the case without deformation, and a few domains with higher density appear locally. From Fig. (7b), the stress has a higher value in the area corresponding to Fig. (7a), and a structure bearing mac roscopic deformation is formed with an increase of fiber density. In the area with higher stress, the direction in the vector of the fiber density changes to 45 or $135 \mathrm{deg}$ as shown in Fig. (6). The local stress induced by macroscopic deformation plays an important role in tissue formation.

\section{CONCLUDING REMARKS}

In this paper, the vector of collagen fiber density was defined by its similarity with the dislocation density tensor. The vector was sensitive to fibrous tissue formation of type I collagen, which is the principal ingredient of skin. The model for the motion of fibroblasts, a group of reactiondiffusion equations generalized by considering a mechanical stimulation, cell migration and chemotaxis, and the rate form of constitutive equation for the fibrous tissue of collagen was formulated. By coupled analyses with the finite element method introduced in these models, the influences of macroscopic deformation on fibrous tissue formation of collagen were examined.

\section{ACKNOWLEDGEMENT}

Authors wish to thank Professor Philippe K. Maini of Oxford University in UK for providing his valuable papers and discussions. We are also grateful to Mr. Masakazu KUME of Hitachi, Co., Ltd., for his assistance with the computations. 


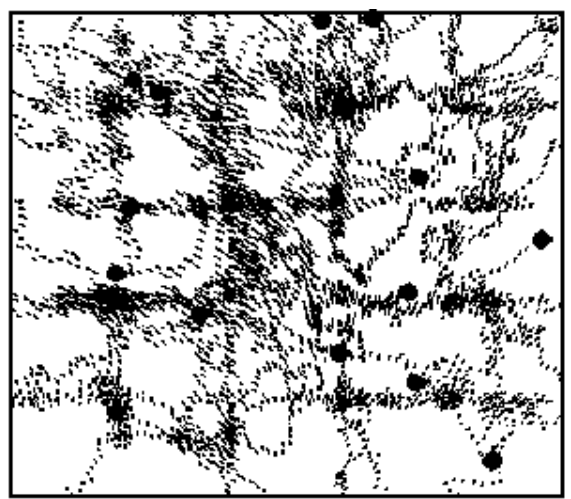

(a) Distribution in direction of fiber for $10 \%$ stretching

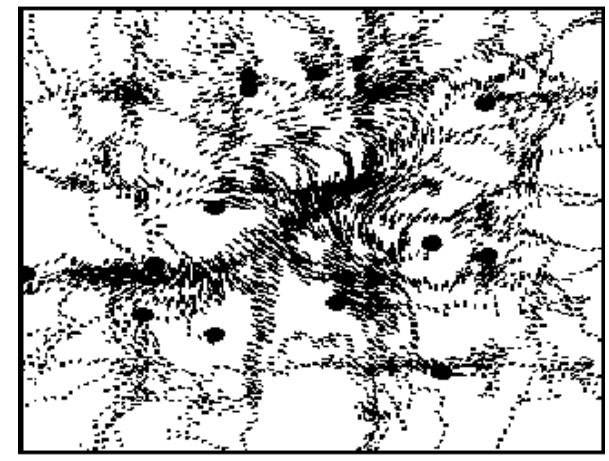

(b) Distribution in direction of fiber for $20 \%$ stretching

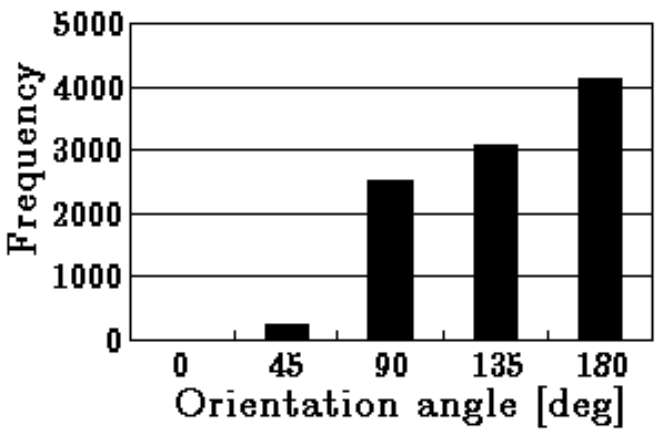

(c) Histogram in orientation angle for $10 \%$ straining

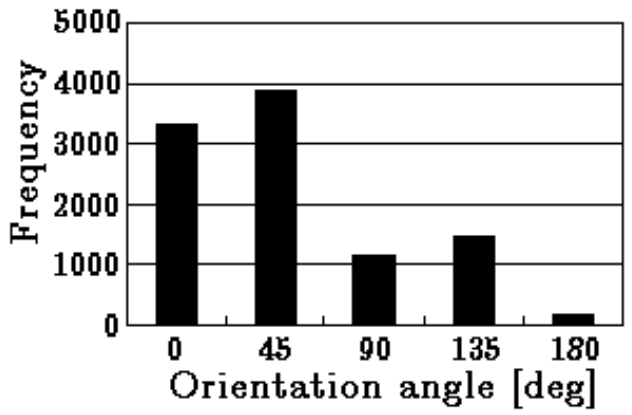

(d) Histogram in orientation angle for $10 \%$ straining

Fig. (6). Computational results on fibrous tissue formation under tensile deformation in horizontal direction

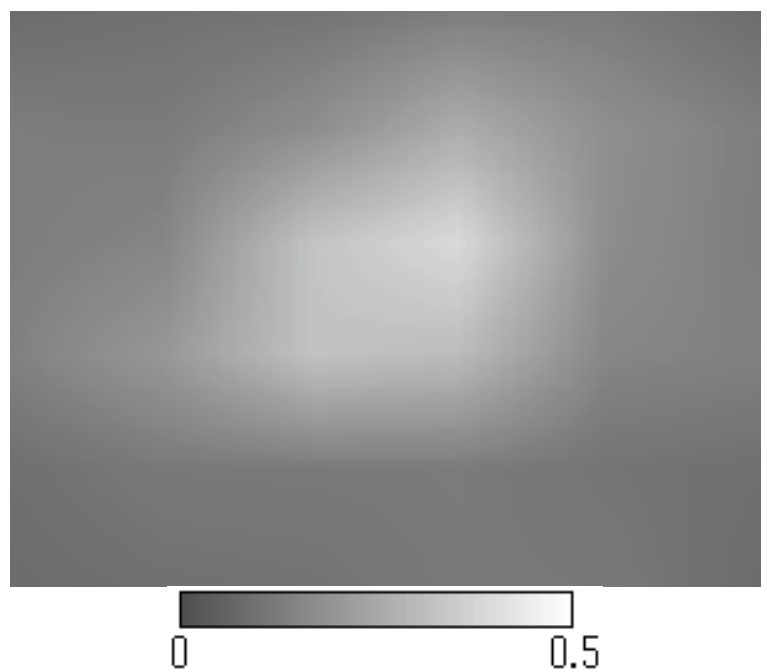

(a) Density of collagen fiber

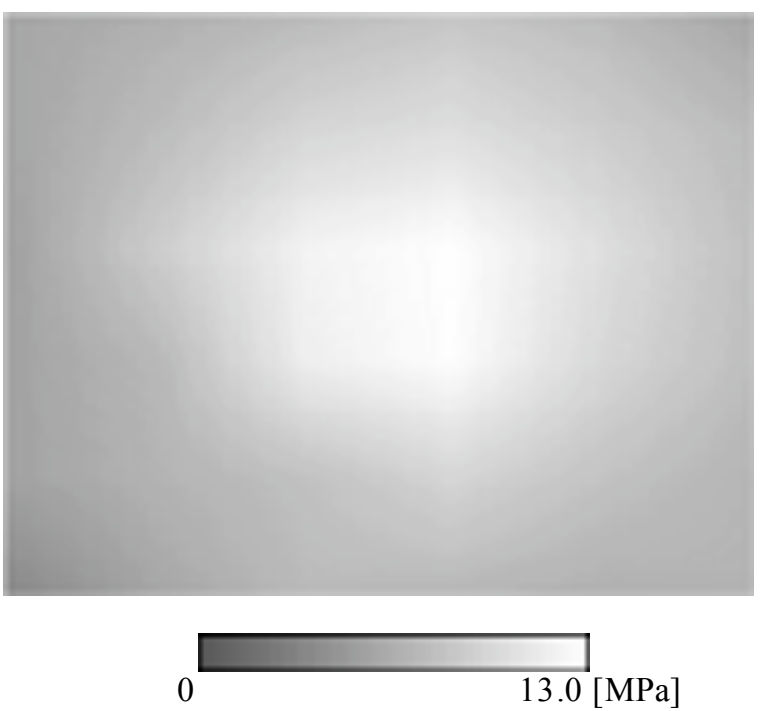

(b) Stress $\sigma_{x x}$

Fig. (7). Distribution of collagen fiber density and stress component in horizontal direction under $20 \%$ straining.

\section{REFERENCES}

[1] Baran R, Maibach HI. Textbook of Cosmetic Dermatology, Third Edition. Informa Healthcare; 2004.

[2] Zern MA, Reid LM. Extracellular Matrix. CRC Press; 1993.

[3] Tsubota K, Adachi T, Tomita Y. Functional adaptation of cancellous bone in human proximal femur predicted by trabecular surface remodelingsimulation toward uniform stress state. J Biomech 2002; 35: 1451-551.

[4] Tezuka K, Wada Y, Kikuchi M. iBone: a reaction diffusion based shape optimization method. Key Eng Mater 2003; 243-244: 601-6.

[5] Eskin SG, Ives CL, McIntire LV, Navarro LT. Response of cultured endothelial cells to steady flow. Microvasc Res 1984; 28: 8794.

[6] Dale PD, Sherratt JA, Maini PK. A mathematical model for collagen fibre formation during foetal and adult dermal wound healing. Proc R Soc Lond 1996; B263: 653-60.

[7] Dallon JC, Sherratt JA, Maini PK. Mathematical modelling of extracellular matrix dynamics using discrete cells: Fiber orientation and tissue regeneration. J Theor Biol 1999; 199: 449-71. 
[8] Takakuda K, Miyairi H. Tensile behaviour of fibroblasts cultured in collagen gel. Biomaterial 1996; 17: 1393-7.

[9] Bellows CG, Melcher AH, Aubin JE. Association between tension and orientation of periodontal ligament fibroblasts and exogenous collagen fibres in collagen gels in vitro. J Cell Sci 1982; 58: 25138.

[10] Mura T. Micromechanics of defects in solids. Martinus Nijhoff: North Holland; 1987.

[11] Bathe KJ. Finite Element Procedures. Prentice-Hall: Englewood Cliffs; 1995.

[12] Wolff J. The law of bone remodeling. Springer: Berlin; 1986.

[13] Zaikov GE. Chemical And Biochemical Physics: New Frontiers. Nova Science Publishers; 2006.
[14] Grodzinsky AJ, Kamm RD, Lauffenburger DA. Quantitative Aspects of Tissue Engineering: Basic Issues in Kinetics, Transport and Mechanics, Principle of Tissue Engineering. Academic Press: California; 1997.

[15] Beckerle MC. Cell Adhesion. Oxford University Press: Oxford; 2002.

[16] Haut RC, Little RW. A constitutive equation for collagen fibers. J Biomech 1972; 5: 423-30.

[17] Adachi E, Takeda Y, Nakazato K, et al. Isolated collagen IV retains the potential to form an 18-nm polygonal meshwork of the lamina densa. J Electr Microsc 1997; 46: 233-41.

(C) Iwamoto et al.; Licensee Bentham Open.

This is an open access article distributed under the terms of the Creative Commons Attribution License (http://creativecommons.org/licenses/by/2.5/), which permits unrestrictive use, distribution, and reproduction in any medium, provided the original work is properly cited. 